\title{
ACL Primary Repair with Bone Marrow Stimulation and Growth Factors. A Review of Literature
}

\author{
Romulus Fabian Tatu, Mihai Hurmuz, Cătălin Adrian Miu \\ XVth Department, "Victor Babeș” University of Medicine and Pharmacy, Timișoara, Romania
}

\section{CORRESPONDENCE}

\section{Mihai Hurmuz}

Str. Munteniei nr. C35 ap. 17

300362 Timișoara, Romania

Tel: +40 740804055

E-mail: hurmuz_mihai@yahoo.com

\section{ARTICLE HISTORY}

Received:

Accepted:
Romulus Fabian Tatu • P-ta Eftimie Murgu nr 2 300041 Timișoara, Romania. Tel: +40 256204400 Cătălin Adrian Miu • P-ța Eftimie Murgu nr. 2, 300041 Timișoara, Romania. Tel: +40 256204400

\begin{abstract}
Anterior cruciate ligament $(\mathrm{ACL})$ ruptures represent a common pathology, especially in young and active patients. Spontaneous repair, although reported in some studies, is altered by local conditions, thus emerges the need to perform reconstruction of the ACL. It is reported that 3,430 primary reconstructions and around 267 revisions are performed yearly in Sweden Some reconstructions result in biological failure, which represents the inability of the graft to incorporate and remodel in order to perform its role as a knee stabilizer. Orthobiology, a new concept that includes growths factors, stem cells, and different scaffolds, could represent a solution to a better outcome of this procedure. This manuscript is a review of different therapeutic strategies used for enabling $A C L$ regeneration, including in vitro ACL-bio-enhanced repair that is currently being developed. Substantial progress is to be expected in the area of ACL surgery.
\end{abstract}

Keywords: $A C L$ reconstruction, growth factors, stem cells, orthobiology

\section{INTRODUCTION}

The anterior cruciate ligament (ACL) represents one of the most important knee stabilizers and one of the most frequently wounded ligaments in young and active persons. It is well known that the ACL has two bundles, the anteromedial one and the posterolateral one. ${ }^{1}$ These two bundles work together to stabilize the knee joint both in flexion and extension. The ACL is the most important stabilizer of the anterior translation of the tibia, but it also helps with rotation and valgus stress. It is an intra-articular ligament with its own synovial membrane; hence, some consider the ligament as extraarticular. When an injury occurs and the ACL is torn, the synovial membrane also ruptures, and the ligament is exposed to synovial fluid, proteolytic enzymes, and hemorrhagic breakdown products. ${ }^{1,2}$ These are the main reasons why spontaneous healing is not possible. Although some studies mention good functional results after conservative treatment, the main accepted treatment nowadays is the surgical reconstruction of 
the ACL. ${ }^{3,4}$ There are many tissue sources that can be utilized to reconstruct the ligament, both auto- and allografts. When talking about autografts, hamstring is reported to be used in about $98 \%$ of cases, but patellar tendon and quad tendon can also be used. ${ }^{5}$ Allograft options include synthetic materials or tissue from tissue banks. The Swedish ACL Register reports an annual rate of 3,430 primary reconstructions of ACL for 2014 with an increasing number each year and a rate of 267 revisions per year. ${ }^{5}$ More than half of the patients are men and the average age is 28 years.

There are certain known problems that lead to failure of a reconstruction, thus making it a complication both for the surgeon and the patient. The main problems regarding failure are technical errors such as tunnel location, graft impingement, inadequate graft dimensions, or graft fixation. ${ }^{6}$ The tunnel location is the primary source of bad outcome, but surgeons strive to be as anatomic as possible, and the results have been improved. Other causes that lead to failure are traumatic injuries and biological failure. The latter is regarded as a lack of ligamentization one year post-implantation, and one has to deal with a lax and atonic graft, and more important, a non-functional reconstruction. Biopsies of the graft reveal marked necrosis, few viable cells, lack of vascularity, and disintegration of collagen. ${ }^{7}$

The ligamentization process includes several steps such as graft necrosis, which happens mainly in the central part of the graft, lasts for about three to four weeks, and is an avascular necrosis. The next step is the revascularization, followed by cell repopulation and proliferation. The graft reaches full perfusion around six to eight weeks, and the cell proliferation occurs mainly from the infrapatellar fat pad and the posterior synovial tissues. The final step is collagen remodeling, finalized around 12 weeks postoperatively. For the collagen production to take place, it needs the previous steps to be completed and to have good mechanical forces applied to it. ${ }^{8}$

The biological failure of a graft is intended to be overcome by regenerative means such as growth factors, stem cells, and even several scaffolds.

\section{GROWTH FACTORS}

Growth factors (GF) can be administered either directly by local delivery, by inserting the cells that produce them at the site of interest, or through gene-modifying therapies. There are a variety of GF, including insulin-like growth factor (IGF), transforming growth factor (TGF- $\beta$ ), platelet-derived growth factor (PDGF), vascular endothelial growth factor (VEGF), epidermal growth factor (EGF), and fibroblast growth factor (FGF) that have been used to improve ligamentous healing capabilities. Growth factors exhibit the ability to stimulate the anabolism of the cells in the ligament, thus improving cell proliferation and extracellular matrix (ECM) deposition, and can also enhance the differentiation of mesenchymal stem cells (MSCs) into fibroblasts. ${ }^{9,10}$

\section{PLATELET-RICH PLASMA}

Platelet-rich plasma (PRP) is gained through centrifugation of whole blood, leading to plasma separation containing thrombocytes, serum proteins including fibrin, and several growth factors (PDGF, VEGF, TGF- $\beta$, and IGF). PRP has a minimum thrombocyte concentration of $1,000,000$ cells per microliter and a 3- to 5-times higher GF and cytokine concentration. ${ }^{11}$

In a review by Andriolo et al. they found 15 studies that used PRP with ACL grafts. Most studies had used hamstring tendons for ACL reconstruction, while a bonetendon-bone (BTB) was used in 4 studies and an allograft in one study. PRP was utilized in the clear majority of the studies to promote graft bone integration and was applied directly on the surface of the tendons used for reconstruction. PRP was activated in 14 studies before intraarticular administration. ${ }^{12}$

Considering the graft maturation process, four studies found results in favor of PRP administration, while two reported no differences. When documenting the graft's integration into the bony tunnels, seven authors found no better outcome in the PRP group. Bony tunnel widening was assessed in three reports, and none revealed a better prognosis with PRP injections. ${ }^{13-15}$ The most important feature of this series of clinical studies was the fact that PRP treatment is a safe procedure with no side effects reported in any study. No infections and no other complication were noted, and two studies even showed improved local healing when administered to the local harvest site of the BTB. ${ }^{16,17}$ The results of this review provided questionable results regarding ACL augmentation and PRP usage. Nevertheless, PRP is a secure procedure providing benefits in the healing mechanism after ACL surgery.

\section{STEM CELLS}

Mesenchymal stem cells are found in the majority of tissues in the body (bone marrow, fat, muscles, and placenta). For the purpose of cellular therapies, MSCs recruited from the bone marrow and fat tissue are the most frequently used. MSCs have the ability of high expansion and self-renewal, and the potential of multi-differentiation. ${ }^{18}$ 
Bone marrow-derived mesenchymal stem cells (BMMSCs) have been researched widely, but adipose-derived stromal cells (ASC) are accessible cells that can replace BM-MSCs. The abundance of tissue and the simplified harvesting process have made the adipose tissue a new source for providing cells for regenerative medicine. ${ }^{19}$ The minimal criteria that define the MSCs set by the International Society for Cellular Therapy include the presence of CD105, CD73, and CD90 surface marker, the lack of CD45, CD34, CD14, CD31, and HLA-DR markers, their capacity to stick to plastic and to differentiate into osteogenic, chondrogenic, and adipogenic lineage. ${ }^{20}$

Kanaya et al. studied from a biomechanical and histological point of view if MSCs can improve the healing of a partial ACL rupture in a rat model. They reported improved outcomes both from biomechanical and histological point of view. ${ }^{21}$

Silva et al. investigated the use of non-cultivated BMMSCs in ACL reconstruction in humans. Unfortunately, no characterization of the aspirate, viability, or numeration was provided. MRI did not detect any differences between BM-MSCs and the control group. ${ }^{22}$

Both Soon et al. and Lim et al. showed, in a rabbit model, that MSCs enhance the graft osteointegration both for autograft and allograft. In both studies the MSCs improved ACL reconstructions providing significantly better results than the control groups. ${ }^{23,24}$

Cheng et al. found better results when researching stem cells derived from the ACL itself in comparison to bone marrow-derived MSCs in ECM production and proliferation rate..$^{25}$

\section{SCAFFOLDS}

Various biomaterials have been presented as potential scaffolds for the ACL. A scaffold is a biological match, and its mechanical features resemble those of the native ACL. The structure of a scaffold has to be biodegradable in order to enable tissue ingrowth and in time to reestablish the structure of a native ACL. ${ }^{26}$ The scaffold must be flexible in the low-tension stresses of everyday motions, but very strong in the larger range strain, in order to ensure knee stability.

Dunn et al. and Bellincampi et al. developed scaffolds made of collagen fibrils and reported that ACL fibroblasts adhere to the scaffold both in vivo and in vitro, but unfortunately at six weeks the combination was disintegrated.27,28

Murray et al. showed that a collagen-glycosaminoglycan compound scaffold maintained cell growth and proved that the human ACL cells can migrate weeks after the rupture into the scaffold. ${ }^{29}$ Panas-Perez et al. developed a collagen-silk complex and concluded that a roughly $50 \%$ composition of silk assures the minimal requirements of a functional ACL in vivo. ${ }^{30}$

Altman and Kaplan managed to twist fiber scaffolds to match the strength of the native human ACL. ${ }^{31}$ Fan et al. showed that a silk ligament scaffold in conjunction with seeded MSCs support ligament repair, and although degradation was present at twenty-four weeks after implantation in a pig model, it still maintained sufficient tensile load. ${ }^{32}$ Electrospinning has been utilized to develop scaffolds for ligament tissue engineering. ${ }^{33}$ This method may be used to create very strong fibers ranging from nanometers to microns in size, allowing a more exact adaptation of the mechanical features of the construct.

\section{FUTURE DEVELOPMENTS}

The era of orthobiology is just starting to develop. New biomaterials and novel techniques for the improvement of cell and scaffold management will be developed. This will involve developing new therapies, based on regenerative medicine. In regards to cellular therapies, the mechanisms involved in such procedures are yet to be fully discovered.

\section{CONFLICT OF INTEREST}

Nothing to declare.

\section{REFERENCES}

1. Kraeutler MJ, Wolsky RM, Vidal AF, Bravman JT. Anatomy and Biomechanics of the Native and Reconstructed Anterior Cruciate Ligament: Surgical Implications. J Bone Joint Surg Am. 2017;99:438-445.

2. Petersen W, Zantop T. Anatomy of the anterior cruciate ligament with regard to its two bundles. Clin Orthop Relat Res. 2007;454:35-47.

3. Costa-Paz M, Ayerza MA, Tanoira I, Astoul J, Muscolo DL. Spontaneous Healing in Complete ACL Ruptures: A Clinical and MRI Study. Clin Orthop Relat Res. 2012;470: 979-985.

4. Roe J, Salmon L, Waller A, Linklater J, Pinczewski L. Spontaneous Healing of the Ruptured Anterior Cruciate Ligament. A Case Series of 21 Patients. Orthop J Sports Med. 2016;4:2325967116S00080.

5. Swedish ACL Register. Annual Report 2014. Available at: https://www artroclinic.se/info/rapport2014en.pdf

6. Samitier G, Marcano Al, Alentorn-Geli E, Cugat R, Farmer KW, Moser MW. Failure of Anterior Cruciate Ligament Reconstruction. Arch Bone Jt Surg. 2015;3:220-240.

7. Ménétrey J, Duthon VB, Laumonier T, Fritschy D. "Biological failure" of the anterior cruciate ligament graft. Knee Surg Sports Traumatol Arthrosc. 2008;16:224-231.

8. Claes S, Verdonk P, Forsyth R, Bellemans J. The "ligamentization" process in anterior cruciate ligament reconstruction: what happens to the human graft? A systematic review of the literature. Am J Sports Med. 2011;39:2476-2483.

9. Rizzello G, Longo UG, Petrillo S, et al. Growth Factors and Stem Cells for the Management of Anterior Cruciate Ligament Tears. Open Orthop J. 2012;6:525-530.

10. Bissell L, Tibrewal S, Sahni V, Khan WS. Growth factors and platelet rich plasma in anterior cruciate ligament reconstruction. Curr Stem Cell Res Ther. 2015;10:19-25 
11. Laver L, Marom N, Dnyanesh L, Mei-Dan O, Espregueira-Mendes J, Gobbi A. PRP for Degenerative Cartilage Disease: A Systematic Review of Clinical Studies. Cartilage. 2016:1947603516670709.

12. Andriolo L, Di Matteo B, Kon E, Filardo G, Venieri G, Marcacci M. PRP Augmentation for ACL Reconstruction. Biomed Res Int. 2015;2015:371746.

13. Mirzatolooei F, Alamdari MT, Khalkhali HR. The impact of platelet-rich plasma on the prevention of tunnel widening in anterior cruciate ligament reconstruction using quadrupled autologous hamstring tendon: a randomised clinical trial. Journal of Bone and Joint Surgery. 2013;95:6569.

14. Vadalà A, Iorio R, De Carli A. Platelet-rich plasma: does it help reduce tunnel widening after ACL reconstruction? Knee Surg Sports Traumatol Arthrosc. 2013;21:824-829.

15. Orrego M, Larrain C, Rosales J. Effects of platelet concentrate and a bone plug on the healing of hamstring tendons in a bone tunnel. Arthroscopy. 2008;24:1373-1380.

16. de Almeida AM, Demange MK, Sobrado MF, Rodrigues MB, Pedrinelli A, Hernandez A J. Patellar tendon healing with platelet-rich plasma: a prospective randomized controlled trial. The American Journal of Sports Medicine. 2012;40:1282-1288.

17. Cervellin M, de Girolamo L, Bait C, Denti M, Volpi P. Autologous plateletrich plasma gel to reduce donor-site morbidity after patellar tendon graft harvesting for anterior cruciate ligament reconstruction: a randomized, controlled clinical study. Knee Surg Sports Traumatol Arthrosc. 2012;20:114-120

18. Jang KM, Lim HC, Bae JH. Mesenchymal Stem Cells for Enhancing Biologic Healing after Anterior Cruciate Ligament Injuries. Curr Stem Cell Res Ther. 2015:10:535-547.

19. Cheng KH, Kuo TL, Kuo KK, Hsiao CC. Human adipose-derived stem cells: Isolation, characterization and current application in regeneration medicine. Genomic Med Biomarkers Health Sci. 2011;3:53-62.

20. Dominici M, Le blanc K, Mueller I, et al. Minimal criteria for defining multipotent mesenchymal stromal cells. The International Society for Cellular Therapy position statement. Cytotherapy. 2006;8:315-317.

21. Kanaya A, Deie M, Adachi N, Nishimori M, Yanada S, Ochi M. Intra-articular injection of mesenchymal stromal cells in partially torn anterior cruciate ligaments in a rat model. Arthroscopy. 2007;23:610-617.
22. Silva A, Sampaio R, Fernandes R, Pinto E. Is there a role for adult noncultivated bone marrow stem cells in ACL reconstruction? Knee Surg Sports Traumatol Arthrosc. 2014;22:66-71.

23. Soon MY, Hassan A, Hui JH, Goh JC, Lee EH. An analysis of soft tissue allograft anterior cruciate ligament reconstruction in a rabbit model: a short-term study of the use of mesenchymal stem cells to enhance tendon osteointegration. Am J Sports Med. 2007;35:962-971.

24. Lim JK, Hui J, Li L, Thambyah A, Goh J, Lee EH. Enhancement of tendon graft osteointegration using mesenchymal stem cells in a rabbit model of anterior cruciate ligament reconstruction. Arthroscopy. 2004;20:899-910.

25. Cheng MT, Liu CL, Chen TH, Lee OK. Comparison of potentials between stem cells isolated from human anterior cruciate ligament and bone marrow for ligament tissue engineering. Tissue Engineering-Part $A$. 2010;16:2237-2253.

26. Proffen BL, Perrone GS, Gordon R. Bridge-Enhanced ACL Repair: A Review of the Science and the Pathway through FDA Investigational Device Approval. Ann Biomed Eng. 2015;43:805-818.

27. Dunn MG, Liesch JB, Tiku ML, Zawadsky JP. Development of fibroblastseeded ligament analogs for ACL reconstruction. J Biomed Mater Res. 1995;29:1363-1371.

28. Bellincampi LD, Closkey RF, Prasad R, Zawadsky JP, Dunn MG. Viability of fibroblast-seeded ligament analogs after autogenous implantation. $J$ Orthop Res. 1998;16:414-420.

29. Murray MM, Spector M. The migration of cells from the ruptured human anterior cruciate ligament into collagen-glycosaminoglycan regeneration templates in vitro. Biomaterials. 2001;22:2393-2402.

30. Panas-Perez E, Gatt CJ, Dunn MG. Development of a silk and collagen fiber scaffold for anterior cruciate ligament reconstruction. J Mater Sci Mater Med. 2013;24:257-265.

31. Altman GH, Horan RL, Lu HH, et al. Silk matrix for tissue engineered anterior cruciate ligaments. Biomaterials. 2002;23:4131-4141.

32. Fan H, Liu H, Toh SL, Goh JC. Anterior cruciate ligament regeneration using mesenchymal stem cells and silk scaffold in large animal model. Biomaterials. 2009;30:4967-4977.

33. Cardwell RD, Dahlgren LA, Goldstein AS. Electrospun fibre diameter, not alignment, affects mesenchymal stem cell differentiation into the tendon/ ligament lineage. J Tissue Eng Regen Med. 2014;8:937-945. 